\title{
Gestión de desechos químicos en laboratorios de la Universidad Nacional
}

José Carlos Mora Barrantes'

Gilberto Piedra Marín 2

Roy Pérez Salazar ${ }^{3}$

Gustavo Chaves Barboza4

David Benavides Ramírez ${ }^{5}$

Fecha de recepción: 22 de marzo del 2011

Fecha de aprobación: 17 de setiembre del 2011

Mora, J; Piedra, G; Pérez, R; Chaves, G; Benavides, D. Gestión de desechos químicos en laboratorios de la Universidad Nacional. Tecnología en Marcha. Vol. 25, No I. Enero-Marzo 2012 Pág 64-69

I. Máster en Ciencias. Químico industrial. Universidad Nacional de Costa Rica.Tel: 22773403 Correo electrónico: jmor@una.ac.cr.

2 Doctor, Químico puro, Universidad Nacional de Costa Rica. Tel: 22775549 Correo electrónico: gilberto.piedra@gmail.com.

3 Máster en Ciencias. Químico industrial. Universidad Nacional de Costa Rica.Tel: 22773547 Correo electrónico: rpere@una.ac.cr.

4 Licenciado, Químico industrial. Universidad Nacional de Costa Rica.Tel: 22773547 Correo electrónico: gustavocb06@gmail.com

5 Licenciado, Químico industrial. Universidad Nacional de Costa Rica.Tel 22773139 Correo electrónico: dbenavid@una.ac.cr. 


\section{Palabras clave}

Desechos químicos, gestión de residuos, salud y medio ambiente.

\section{Resumen}

Durante el 2009 se realizó un diagnóstico de la gestión de desechos químicos en la Universidad $\mathrm{Na}$ cional, tanto en laboratorios de docencia como de investigación. Para recopilar la información: I) se aplicó un cuestionario, una entrevista y se hizo una visita (inspección) a los laboratorios; 2) se realizaron inventarios de desechos químicos; 3) se efectuaron sesiones de trabajo con funcionarios encargados de la gestión universitaria; y 4) se visitó a funcionarios de instituciones públicas y privadas encargados de la gestión de desechos.

Con el presente trabajo, se identificaron las condiciones actuales de manipulación y disposición final de los desechos químicos en la Universidad Nacional, así como los procedimientos o gestiones administrativos que ejecutan las autoridades universitarias para su adecuada manipulación.

Se concluye que para una gestión adecuada de desechos químicos en la Universidad Nacional es necesario formular e implementar una normativa, a nivel nacional e institucional, enfocada al quehacer universitario, así como la creación de una oficina que se encargue de coordinar todas las actividades y procedimientos relacionados con el manejo de los desechos químicos.

\section{Key words}

Chemical wastes, waste management, health care and environment.

\begin{abstract}
During year 2009, a diagnostic regarding chemical waste management in teaching and research laboratories of the Universidad Nacional, was carried out. In order to gather such information they were utilized: I - an interview, a questionnaire and an inspection visit to the laboratories, 2- the generation of chemical waste inventories, 3- work sessions with university management authorities and 4-visits with waste-management personnel of public and private institutions.

This study allowed to identify the actual conditions for the manipulation and final disposal of chemical wastes at Universidad Nacional, as well as the existing management procedures executed by university authorities for handling them appropriately.
\end{abstract}

As a conclusion, in order to conduct an adequate chemical waste management at Universidad Nacional it is necessary to formulate regulation, at the institutional and national levels, focused on university activities, as well as the establishment of an university office in charge of all the chemical waste management activities and procedures. 


\section{Introducción}

Para poder sostener la demanda actual de productos y servicios por parte de las sociedades, ha sido forzosoaumentar los niveles de producción a nivel mundial, lo cual ha traído como consecuencia un aumento en los volúmenes de residuos generados que, en muchos casos, incluyen la presencia de materiales peligrosos,lo que ha hecho necesaria una adecuada gestión de los desechos, cuyo objetivo primordial sea la prevención y minimización, de manera que permitan disminuir el riesgo a la salud, a la propiedad y al ambiente (Martínez, 2005).

\section{Etapas para el manejo de desechos peligrosos}

Un adecuado sistema de manejo de desechos peligrosos incluye las etapas de generación, acumulación, almacenamiento, transporte, tratamiento y disposición final.

Con respecto a la generación de desechos, se define como el ente generador aquel que produce uno o más desechos peligrosos como resultado de su actividad; es obligación del ente generador realizar los esfuerzos necesarios para reducir al máximo su producción (Decreto 2700I, 1998).

La acumulación es el proceso de llenado de recipientes para la colecta de los desechos producidos. Esta acción debe llevarse a cabo lo más cerca posible del sitio de generación y se debe contar con condiciones adecuadas para garantizar la seguridad de los trabajadores, de la propiedad y del ambiente, condiciones que, además, deben cumplirse durante el almacenamiento de los desechos.

El almacenamiento es la fase posterior a la acumulación. En esta etapa se conservan los desechos debidamente empacados y embalados para su posterior tratamiento o disposición final. El almacenamiento se lleva a cabo en un sitio externo al de generación, normalmente en una bodega exclusiva para el almacenamiento de desechos (Decreto 2700 I, 1998).

El tratamiento de desechos es el proceso de transformación del desecho; el objetivo del tratamiento es reducir el volumen del desecho y disminuir la peligrosidad del mismo. Dentro de los procesos de tratamiento están: el reciclaje, el reuso, la estabilización y la solidificación, así como los tratamientos fisico- químicos, biológicos y térmicos, entre otros (Decreto 2700 I, 1998).

La etapa de disposición final se refiere a la descarga, inyección, deposición, lanzamiento y/o colocación de cualquier desecho peligroso (previamente tratado) (Decreto 2700I, 1998). Dicha disposición debe hacerse de manera que el desecho, o cualquier constituyente del mismo que entre al ambiente, no acarree ningún tipo de problema para este. Entre los métodos de disposición final se encuentran el relleno sanitario de seguridad, el encapsulamiento, la incineración y la exportación a países desarrollados (Martínez, 2005).

\section{Jerarquías en el manejo de desechos}

En la figura I se muestra el sistema de Jerarquía en la gestión de desechos, que está compuesto por cuatro principios.

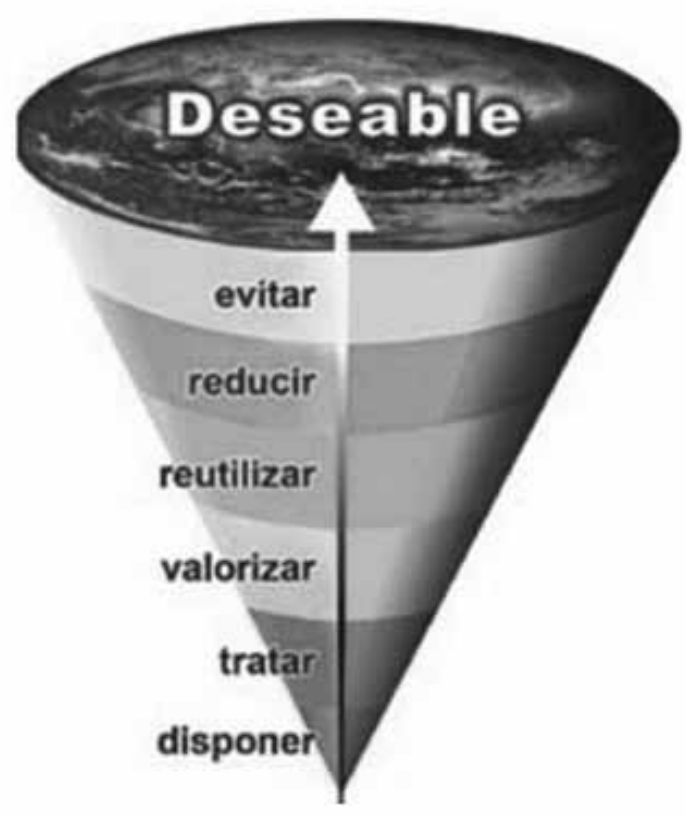

Figura I. Jerarquía en la Gestión de Residuos (Martínez, 2005).

El primero de ellos, prevención y minimización de desechos, consiste en evitar la generación de residuos descle la fuente; es una etapa dirigida primordialmente a la autogestión y su fin único es no generar o minimizar la cantidad de desecho a producir. Esta etapa incluye acciones como la incorporación de 
Cuadro I.Aspectos relacionados con la gestión de desechos químicos en los laboratorios de investigación (I) y docencia

(D) de la Universidad Nacional

\begin{tabular}{|c|c|c|c|}
\hline \multirow[t]{2}{*}{ Etapas } & \multirow[t]{2}{*}{ Aspecto evaluado } & \multicolumn{2}{|c|}{$\begin{array}{l}\text { Número de laboratorios que } \\
\text { respondieron al instrumento } \\
\text { de evaluación }\end{array}$} \\
\hline & & $I(n=48)$ & $D(n=4)$ \\
\hline \multirow[b]{4}{*}{ Generación } & Generación de desechos químicos & 48 & 4 \\
\hline & Implementación de procesos de minimización y prevención & 10 & 0 \\
\hline & Conocimiento de la tasa de generación mensual & 5 & 0 \\
\hline & $\begin{array}{l}\text { Conocimiento de la fuente de generación (para el caso de desecho } \\
\text { acumulado) }\end{array}$ & 10 & 0 \\
\hline \multirow{6}{*}{ Segregación } & $\begin{array}{l}\text { Los puntos de acumulación de los desechos se ubican en un área } \\
\text { diferente al sitio de generación }\end{array}$ & 2 & 0 \\
\hline & $\begin{array}{l}\text { Los puntos de acumulación en el laboratorio cuentan con las } \\
\text { condiciones de seguridad necesarias para evitar accidentes }\end{array}$ & 5 & 0 \\
\hline & $\begin{array}{l}\text { Los desechos químicos se almacenan de acuerdo con criterios de } \\
\text { compatibilidad química, evitando reacciones no deseadas }\end{array}$ & 10 & 0 \\
\hline & $\begin{array}{l}\text { Los recipientes utilizados para contener los residuos son compatibles } \\
\text { con el desecho almacenado }\end{array}$ & 10 & 0 \\
\hline & $\begin{array}{l}\text { Las etiquetas contienen información relativa al desecho (fecha de } \\
\text { acumulación, propiedades físico-químicas, riesgos y peligros) }\end{array}$ & 5 & 0 \\
\hline & $\begin{array}{l}\text { Se hace una supervisión periódica del estado de los desechos (control } \\
\text { de T, pH, estado de etiquetas, recipientes, detección de fugas, derrames, } \\
\text { etc.) }\end{array}$ & 5 & 0 \\
\hline $\begin{array}{l}\text { Almacenamiento } \\
\text { temporal }\end{array}$ & $\begin{array}{l}\text { Los desechos se mantienen acumulados en espera de una solución } \\
\text { institucional }\end{array}$ & 30 & 0 \\
\hline \multirow{5}{*}{$\begin{array}{l}\text { Tratamiento } \\
\text { y } \\
\text { disposición final }\end{array}$} & Los residuos se descargan directamente a la pileta & 14 & 6 \\
\hline & $\begin{array}{l}\text { Los residuos se descargan a la pileta luego del tratamiento físico- } \\
\text { químico previo }\end{array}$ & 2 & 0 \\
\hline & Se reutilizan / recuperan los desechos & 5 & 0 \\
\hline & $\begin{array}{l}\text { Los residuos se acumulan en el laboratorio luego de tratamiento físico- } \\
\text { químico }\end{array}$ & 2 & 0 \\
\hline & Los desechos son tratados por empresas externas & 5 & 0 \\
\hline
\end{tabular}

I= laboratorios de investigación; $\mathrm{D}=$ Laboratorios de docencia; n=número de laboratorios.

buenas prácticas de operación, cambios en tecnología, cambios de materia prima, uso de materiales más durables, modificaciones en los procesos de adquisición, cambios en las condiciones del proceso (flujos, temperatura, presión, tiempo de residencia), etc. (Martínez, 2005).

El segundo principio es el aprovechamiento y valorización de residuos, que involucra el reuso y la recuperación de los materiales con el fin de lograr un mayor ciclo de vida de los materiales. El objetivo principal de este principio es extender el uso del desecho, al reducir la necesidad de producir y procesar nuevos productos, de manera que se genere menos contaminación ambiental (Martínez, 2005).
El tratamiento que se le dé al residuo constituye el tercer principio de la jerarquía de gestión de desechos. Esta opción debe involucrar procesos de transformación amigables con el ambiente y con metas específicas para disminuir el volumen y el peligro de los desechos (Martínez, 2005).

La última alternativa que se debe tomar en cuenta en la gestión de los desechos es la disposición final de los mismos. De acuerdo con este cuarto principio, la cantidad de residuos en esta etapa deber ser mínima y su depósito final debe incorporar tanto aspectos tecnológicos como económicos (Martínez, 2005). 


\section{Gestión de desechos químicos en centros universitarios}

Las universidades son instituciones que presentan problemas muy específicos vinculados con el manejo de sustancias peligrosas y de sus desechos, ya que cuentan con laboratorios muy diversos los cuales generan un amplio espectro de desechos (Mooney, 2004).

La búsqueda de soluciones para la gestión de los desechos en universidades enfrenta desafíos como: I) existencia de normativas estatales confusas y, con respecto a algunos aspectos, en algunas cosas contradictorias para en el ambiente universitario, 2) la necesidad de cambio de conducta y consumo, 3) la descentralización de una estructura organizacional que lidere, en forma conjunta, los temas de salud ocupacional y seguridad ambiental en los centros universitarios (Mooney, 2004).

Diferentes autores, organizaciones y universidades se han pronunciado con relación a esta problemática y han enfocado sus esfuerzos para encontrar soluciones que permitan prevenir la generación de desechos, y que su disposición final se realice de forma óptima en beneficio de la salud y del ambiente. Algunas de estas organizaciones son la Prensa Académica Nacional de Estados Unidos (National Academic Press), la Sociedad Americana de Química (American Chemical Society, ACS) y la Agencia de Protección Ambiental (Evironmental Protection Agency, EPA) de Estados Unidos (Reinhardt, 2009).

Recientemente, EPA y la ACS se han asociado para fomentar investigaciones que incorporen el concepto y principios de la química verde en los programas de investigación universitaria y en el currículo de los académicos (Mooney, 2004). Entre los principios de la química verde están: diseñar reactivos y productos químicos más seguros, crear síntesis químicas menos peligrosas, utilizar materias primas renovables, aumentar el rendimiento energético de reacciones y minimizar el potencial de accidentes, entre otros (Anastas, Wood, Masciangioli, McGowan y Ruth, 1998).

\section{Metodología}

El análisis de la gestión de desechos químicos se enfocó en las unidades académicas que usan sustancias químicas para las actividades de docencia e inves- tigación en los Campus Omar Dengo y Benjamín Núñez. El estudio se realizó durante el 2009.

La población en estudio se seleccionó con base en información recolectada en la Dirección de Investigación de la Vicerrectoría Académica, así como de la Proveeduría Institucional. Los laboratorios y bodegas incluidos en el estudio conforman, aproximadamente, el $80 \%$ de los sitios que manipulan reactivos $y / 0$ productos químicos en las facultades de Ciencias Exactas y Naturales, Ciencias de la Tierra y el Mar, y Ciencias de la Salud.

Para conocer el estado actual del manejo de los desechos: I) se aplicó un cuestionario, una entrevista y se realizó una visita a los sitios, 2) se efectuaron inventarios de desechos químicos, 3) se celebraron sesiones de trabajo con funcionarios encargados de la gestión universitaria y 4) se visitó a funcionarios de instituciones públicas y privadas, como el Ministerio de Salud, el Ministerio de Ambiente y Energía y Telecomunicaciones, el Instituto Tecnológico de Costa Rica, HOLCIM, etc.

\section{Resultados y discusión}

En el cuadro I se muestra un resumen de los resultados obtenidos en la evaluación de la gestión de los desechos químicos en los laboratorios de la UNA. La información del cuadro se refiere a los procedimientos o acciones desarrollados por el personal de los laboratorios durante las etapas de generación, acumulación, almacenamiento, tratamiento y disposición final de los desechos químicos.

De acuerdo con el cuadro I, existen laboratorios cuyo personal es consciente de la gestión de sus desechos, desde la generación hasta su disposición final, por lo que se incorporan acciones como modificaciones en los procesos, substitución de reactivos químicos, control y supervisión de los desechos generados, y adecuación de los sitios para su almacenamiento; no obstante, la mayoría de los laboratorios de la UNA requiere mejorar las acciones realizadas durante las etapas de gestión de los desechos.

El diagnóstico permitió establecer la anuencia del personal de los laboratorios a incorporar mejoras en las condiciones de manipulación y disposición de los desechos.

Los funcionarios expresaron, además, la necesidad de realizar procesos de capacitación en el manejo de 
desechos químicos, especialmente con respecto a las acciones de prevención, minimización, identificación y acumulación de los mismos.

Adicionalmente, se recomienda centralizar la gestión de los desechos en un departamento o programa institucional, en vez de esfuerzos aislados llevados a cabo por grupos de investigación o programas de docencia, que pueda brindar un eficiente control y seguimiento a la disposición final de los residuos químicos.

\section{Conclusiones}

En materia de gestión de desechos en instituciones de enseñanza superior, no existe a nivel nacional reglamentación específica que incorpore las acciones recomendadas por la Sociedad Americana de Química (ACS), la Prensa Académica Nacional (National Academic Press) y la Agencia de Protección Ambiental de Estados Unidos (EPA), así como de universidades de países desarrollados. La regulación nacional en torno a la disposición de desechos está dirigida a la industria química y no incorpora sistemas de clasificación química, condiciones de uso o protocolos para la gestión adecuada de desechos provenientes de actividades de docencia o investigación.

Con respecto a la gestión de desechos químicos en la Universidad Nacional, se concluye que esta actividad no ha sido coordinada por un departamento o programa institucional centralizado que permita articular todos los diferentes requerimientos y necesidades para manipular y disponer, de forma segura, de los desechos; esta deficiencia genera, entre otras cosas, que la mayoría de las unidades académicas no contemple en sus planes operativos anuales la necesidad de mejorar la infraestructura, el equipo y los materiales de seguridad que permitan optimar la gestión de residuos químicos en la institución, ni tampoco incorporen procesos ambientalmente sostenibles en procedimientos analíticos, como, por ejemplo, la implementación de conceptos de química verde.

No obstante, se debe destacar que la presente administración ha mostrado interés y compromiso por mejorar las condiciones actuales en los laboratorios al invertir en equipo, materiales, infraestructura y procesos de capacitación. La creación del Programa UNA Campus Sostenible y la contratación de un Regente Químico han sido fundamentales para la generación de iniciativas destinadas a lograr la adecuada gestión de desechos. Existe, en general, un compro- miso por parte de los coordinadores de laboratorios para mejorar las condiciones de trabajo, así como para incorporar y cumplir con las directrices que en su momento se establezcan para la protección del ambiente.

\section{Agradecimientos}

Al personal académico y administrativo de la Universidad Nacional.

\section{Bibliografía}

Anastas, P.; Wood, F.; Masciangioli, T.; McGowan, E. \& Ruth, L. (Eds.) (2007). Exploring Opportunities in Green Chemistry and Engineering Education:A Workshop Summary to the Chemical Sciences Roundtable. Washington D.C.: National Academy of Sciences.

Ministerio de Ambiente y Energía [MINAE]. (1998). Decreto 2700 I Reglamento para el manejo de los desechos peligrosos. La Gaceta.

Díaz, N. (2000). Manual de gestión de los residuos especiales de la Universidad de Barcelona. Barcelona: Publicacions de la Universitat de Barcelona.

Imbriosi, D.; Moraes, J.; Soares S.; da Fonseca, S.; Monteiro, H.; East Ponce, G.; Furtado, J., ... Fernandes, P. (2006). Management of Chemical Residues in Universities: Assessing the University of Brasilia. Quim, Nova, 29(2), 404409.

Martínez, J. (2005). Guía para la gestión integral de residuos peligrosos. Montevideo: Centro Coordinador del Convenio de Basilea para América Latina y el Caribe.

Monz, D. \& Fiona, M. (2006) EPA's Proposed Academic Laboratories Rule: A More Flexible Approach to the Management of the Hazardous Waste. Elsevier Inc.

Mooney, D. (2004). Effectively Minimizing Hazardous Waste in Academia: The Green Chemistry Approach. Chemical Health and Safety, II (3) 24-28.

Prudent practice for Handling Hazardous Chemicals in Laboratories. (1995). Washington D.C.: National Academy Press

Pipitone, D. (|99|). Safe Storage of Laboratory Chemicals (2 ed.). New York: John Wiley \& Sons Inc.

Pongráez, E.; Phillips, P. \& Keiski, R. (2004, June). From Waste Minimization to Resources Use Optimization: Definition and Legislative Background. Proceeding of the Waste Minimization and Resources Use Utilization Conference, University of Oulu, Finland.

Reinhart, P. (2009). An Update of Prudent Practices in the Laboratory: Handling and Disposal of Chemicals. Washington D.C.: National Academies Press. 